\title{
Des liens entre naturisme et végétalisme : de la communauté végétalienne du Monte Verita à l'influence du naturisme et végétarisme allemand en France
}

\author{
Bernard Andrieu ${ }^{1}$ \\ Guillaume Robin ${ }^{2}$
}

\author{
« Je vivais nu et éveillé, tel un cerf dans son bocage de \\ rocaille. » \\ Hermann Hesse \\ Tiré de Dans les rochers - Notes d'un homme de la nature
}

\begin{abstract}
Ainsi se promenait Hermann Hesse, nu dans les bois, de hutte en hutte, alternant des périodes de jeûne et une alimentation à base de légumes crus et de fruits lorsqu'il se rendit pour la première fois en 1907 dans le Tessin pour rejoindre la colonie du Monte Verita près d'Ascona, en Suisse, afin d'y effectuer une cure de quatre semaines dans le but de guérir sa dépression et son alcoolisme. Un an après, il publia un essai intitulé Dans les rochers - Notes d'un homme de la nature où il retranscrit avec distance sa vie au milieu de la communauté végétarienne du Monte Verita. Il y expérimenta le confort spartiate des huttes d'air et de lumière et le mode de vie alternatif et ascétique de la colonie basée notamment sur les principes du végétalisme ${ }^{4}$. Hesse raconte :
\end{abstract}

Quelques années, le Docteur Knölge avait fait venir le printemps et le début de l'été dans l'une des nombreuses auberges végétariennes sympathiques du lac Majeur. Il avait rencontré beaucoup de gens dans ces lieux et s'était habitué à beaucoup de choses, la marche pieds nus et les apôtres aux cheveux longs, les fanatiques du jeûne et les gourmands végétariens. Il y avait des végétariens, des végétarianiens, des végétabilistes, des crudivores, des frugivores et des mixivores (sic). Le médecin lui-même faisait partie, selon l'expression des initiés, des mixivores. ${ }^{5}$

A en juger par la multiplicité des termes employés ci-dessus par Hesse, l'auteur laisse entendre qu'il y avait au Monte Verita non pas un courant végétarien mais différentes pratiques pouvant aller du végétarisme soft à ce que l'on appellerait aujourd'hui le véganisme (qualifié à l'époque de végétabilisme), lequel pouvait prendre des formes extrêmes comme le crudivorisme ou le fruitarisme. Hermann Hesse fait l'expérience dans ce « coin perdu » de la nudité du corps exposée à la chaleur du soleil et à la fraîcheur du vent et la décrit comme une immersion dans une existence à la fois minérale et végétale :

$\mathrm{Au}$ total, je suis resté sept jours sans manger. Pendant ce temps, ma peau partait en lambeau et se renouvelait, je m'habituais à vivre nu, couché à même le sol, le corps exposé au soleil, au chaud et au froid. Alors que je pensais que j'allais succomber, je suis devenu ferme et coriace (....). Les nuits, je les passais bientôt dans la cabane, bientôt à

\footnotetext{
1 , Professeur à l'Université Paris Descartes Laboratoire I3SP (EA 3625), Université Paris-Descartes GDRI 836 CNRS \& associé UMR 6872 ADES Cnrs/AMU, bernard.andrieu@parisdescartes.fr
}

2 Maître de conférence à l'Université Paris Descartes Laboratoire I3SP (EA 3625)

${ }^{3}$ Hesse, Hermann, «In den Felsen. Notizen eines Naturmenschen » (récit autobiographique), 1907. Lire à ce sujet : Radermacher, Martin, 6-2011, « Hermann Hesse - Monte Verità, Wahrheitssuche abseits des Mainstreams zu Beginn des 20. Jahrhunderts.

https://journals.openedition.org/zjr/710 (dernier accès 16.09.2019)

${ }^{4}$ Le terme de veganisme serait anachronique dans la mesure où il doit son apparition à l'anglais Donald Watson, lors de la création de la «vegan society » en 1944, issue d'un schisme avec la « vegetarian society », prônant par ce terme un végétarisme radical.

${ }^{5}$ Hesse, Hermann Hesse, Weltverbesserer, Sämtliche Erzählungen 1910-1918 (Suhrkamp Taschenbuch): "Manche Jahre hatte Doktor Knölge die Zeit des Frühlings und Frühsommers in einer der vielen freundlichen Vegetarierpensionen am Lago Maggiore hingebracht. Er hatte vielerlei Menschen an diesen Orten kennengelernt und sich an manches gewöhnt, an Barfussgehen und langhaarige Apostel, an Fanatiker des Fastens und an vegetarische Gourmands. Da gab es Vegetarier, Vegetarianer, Vegetabilisten, Rohkostler, Frugivoren und Gemischtkostler. Der Doktor selbst gehörte nach dem Sprachgebrauch der Eingeweihten zu den Gemischtkostlern." 
l'extérieur, au bord de l'eau aussi. Souvent, je somnolais pendant des heures jusqu'à ce que ma soif me réveille. Souvent, je passais des heures allongé dans un état de semi-conscience, je voyais la lumière et l'ombre changer et j'entendais les petits bruits de cette terre de désolation, sans les respecter et sans me rendre compte de ce que je voyais ou j'entendais. Parfois, j'avais l'impression de devoir me pétrifier, m’enraciner et replonger dans une existence végétale ou minérale ${ }^{6}$.

Si Hesse prit ses distances envers ce mode de vie spartiate qu'impliquait ce retour à la nature, il souligne en même temps par son témoignage l'articulation du naturisme et du végétalisme, deux courants nés à la même époque dans la mouvance de de la Lebensreform. Il s'agira d'analyser dans cet article la nature de ces liens en portant dans un premier temps une attention particulière à la communauté végétalienne du Monte Verita et aux initiatives qui l'ont précédé et, dans un second temps, à l'influence du naturisme et du végétalisme allemand en France. Le Monte Verita ne constitue pas en tant que tel la première communauté qui ait prôné et institué (du moins au début) un végétarisme radical. Au seuil du 20ème siècle, le végétarisme en Allemagne ${ }^{7}$ puisait déjà dans un important vivier de penseurs et d'associations et s'appuyait même sur un tissu économique constitué de restaurants végétariens, de coopératives et de Reformhäuser ou magasins de produits naturels.

Il est assez difficile d'estimer le nombre de sympathisants de la cause végétarienne à la fin du 19ème siècle mais des éléments historiques donnent à penser que les idées des végétariens étaient déjà bien répandues en Allemagne comme en Autriche au début du 20ème siècle. Ainsin, lors du Salon de l'Industrie Manufacturière en 1896, événement organisé à Berlin au coeur de Treptower Park et qui attira près de quatre mille exposants et environ 7 millions de visiteurs, un dénommé Joseph Scharberg fut à l'origine du pavillon végétarien où un petit-déjeuner fut organisé pour une centainescde participants, fonctionnaires, représentants de la presse, exposants et professeurs d'université. En Autriche, des sources attestent également de la pénétration des idées végétariennes.

Lors de la «soirée des fous » (Narrenabend), l'un des moments forts du carnaval du Vienne, les membres de la Männergesangerein de 1881 se déguisaient en végétariens à la fin du 19ème siècle ${ }^{8}$, déambulant dans des tuniques amples et des rameaux de cerisiers dans les cheveux pour les singer. Il s'agissait, sur le mode du détournement, de tourner en dérision les végétariens en imitant leur costume, de pointer du doigt leur mode de vie. En 1911, toujours à Vienne, le directeur du spectacle de variétés Leopold Rühl, lors du «Bal des Crapules » (Lumpenball) se vit décerner un prix pour son costume de végétarien avec lequel il égaya les invités du bal en « cherchant à les convertir au fourrage vert et en craquant devant la moindre friandise verte à grignoter ${ }^{9}$. Le fait de prendre les végétaliens dès la fin du 19ème siècle comme une source de plaisanterie lors du carnaval, est un indice qui souligne la portée des thèses végétariennes à l'époque. Comme le souligne Judith Baumgartner, les idées végétariennes commencèrent à être diffusées après la révolution de 1848, où les révolutionnaires déçus qui avaient survécu aux incarcérations ou ne s'étaient pas exilés, tentèrent, devant l'échec politique du mouvement, de changer la société par d'autres voies. L'expansion du végétarisme est à mettre au compte de plusieurs penseurs.

\section{Des premiers penseurs du végétarisme aux Reformhäuser}

Le 21 avril 1867, le théologue luthérien Edouard Baltzer fonda dans une petite commune de Thuringe, Nordhausen, «l'Association pour un mode de vie naturel» (Verein für natürliche Lebensweise).

\footnotetext{
${ }^{6}$ Ibid. „Im ganzen blieb ich sieben Tage ohne Essen. Während dieser Zeit schälte und erneuerte sich meine Haut, ich gewöhnte mich an Nacktsein, hartes Liegen, an Sonnenhitze und kalten Nachtwind. Während ich zu erliegen glaubte, wurde ich fest und zäh [. . .]. Die Nächte brachte ich bald in der Hütte, bald draußen in der Nähe des Wassers zu. Oft schlummerte ich stundenlang, bis der Durst mich weckte. Oft lag ich stundenlang bei halbem Bewußtsein, sah Licht und Schatten wechseln und hörte die kleinen Geräusche der Einöde, ohne ihrer zu achten und mir über das, was ich sah und hörte, Rechenschaft zu geben. Manchmal schien es mir, als müsse ich erstarren, Wurzeln schlagen und in ein pflanzliches oder mineralisches Dasein zurücksinken.“

7 Cluet, Marc, Sirost Olivier eds., 2018, Monte Verità. Communautés d'expériences du corps et de retours à la nature, Cahiers d'Histoire culturelle, n²9, Université de Tours.

${ }^{8}$ « Der Narrenabend des Wiener Männergesang-Vereins », in: Die Presse, 26.1.1881: 14-15, cité par Pack, Birgit : «Ich geh’ im Fasching als Vegetarianer », in « Vegetarisch in Wien um 1900 », 2017. Cf. veggie.hypotheses.org.

${ }^{9}$ Großer Lumpenball, in: Deutsches Volksblatt, 3.3.1911: 20.
} 
Celle-ci était dotée de sa propre revue et fut rebaptisée deux ans plus tard en « Association Allemande pour un mode de vie conforme à la nature » (Deutscher Verein für naturgemässe Lebensweise). Selon Baltzer, le mode de vie végétarien devait contribuer à rendre caduque l'élevage basé sur la propriété terrienne. La terre, de même que l'air et le soleil, ne devrait selon lui n'appartenir à personne mais être partagée par la communauté ; la propriété, elle, est source de misère ${ }^{10}$. La terre est considérée par Baltzer comme le bien de tous et les dépenses qualifiées d' «improductives », telles l'élevage d'animaux ou les dépenses militaires, doivent être abolies et laisser place à un mode de vie en accord avec la terre. Des initiatives similaires à celles de Baltzer apparurent, comme la création quasi concomitante par Gustav Struve de la Stuttgarter Vegetarierverein (1868) et de "l'Association Allemande pour un mode de vie harmonieux » (Deutscher Verein für Harmonische Lebensweise) en 1879. C'est dans le café-auberge végétarien Pomona à Leipzig que se retrouvèrent l'association fondée par Baltzer, l'Association Allemande pour un Mode de Vie harmonieux et d'autres associations locales pour donner naissance à la Fédération Allemande des Végétariens (Deutscher Vegetarierbund). La fédération comptait à sa création près de 400 membres et le nombre s'éleva en 1904 à 1500. Il faudra attendre 1908 pour que se tienne à Dresde le premier congrès international des végétariens, où fut fondée l'International Vegetarian Union.

L'avocat et publiciste Gustav Struve faisait partie des révolutionnaires de 1848 et fut inspiré par les idées du Français Jean Antoine Gleizes (1773-1843) et son ouvrage Thalysie ou La nouvelle existence $^{11}$. Gleizes, un érudit et écrivain, répugnant très vite aux vivisections pratiquées pendant ses études de médecine, se mit à interdire la chasse sur ses terres et à étudier dans sa propriété des Pyrénées ariégeoises les principes de l'alimentation végétarienne. Il y édifia une théorie basée sur l'harmonie avec la nature impliquant de renoncer à un régime carné au profit d'un régime végétal conforme selon lui à la morale. Inspiré par les réflexions de Gleizes, Struve créa son association à son retour d'exil aux Etats-Unis et rédigea en 1869 un ouvrage intitulé Pflanzenkost - Die Grundlage einer neuen Weltanschauung («La nourriture à base de plantes - base d'une nouvelle vision du monde $\gg$ ).

L'un des autres pionniers du végétarisme était Theodor Hahn (1824-1883). Ce dernier avait souffert pendant longtemps de problèmes de santé et avait réussi à se soigner grâce à une cure de balnéothérapie et un régime sans viande, à base de légumes crus et de pain complet. Il raconta sa guérison dans un livre intitulé Le régime approprié » (Die Naturgemässe Diät) et diffusa ses idées en éditant la revue spécialisée Der Naturarzt («Le médecin de la nature »). Hahn comptait parmi ses adeptes Eduard Baltzer dont l'association servit de camp de base pour la diffusion des idées végétariennes.

En 1896, un autre ouvrage rencontra un écho important. Ce fut le livre d'Adolf Just, « Retour à la nature » (Kehrt sur Natur zurück), réédité par douze fois et publié jusqu'à 50000 exemplaires jusqu'en 1930. Libraire de profession, Just, tombé malade, s'était tourné vers des méthodes naturelles de guérison. Il ouvrit en 1895 un centre de santé à Jungborn, dans le Harz, où il reçut notamment Kafka comme patient, avant d'en créer un autre au sortir de la Première Guerre mondiale, nommé la «Société de Guérison par la Terre » (Heilerde-Gesellschaft). L'institut thermal de Jungborn proposait à ses patients une cure sans alcool et sans viande, à base de légumes crus, de fruits, de baies, de noix et de céréales de blé complet. Ce lieu de repos prévoyait aussi des espaces pour prendre des bains et se détendre, nus, au soleil. Just dénonçe dans son ouvrage la cruauté dont sont victimes les animaux en soulignant que l'être humain n'a ni les dents, ni l'intestin d'un carnivore et que la majorité serait bien incapable de tuer un animal de ses propres mains. Il répudie les massacres d'animaux en tant que forme de violence mais aussi car la viande, selon lui, est cause de maladies. Si dans son institut, naturisme et végétarisme sont liés, il n'est pas cependant question d'un véritable retour à la nature

\footnotetext{
${ }^{10}$ Ideen zur sozialen Reform, Nordhausen, 1873, p. 14: «Der Gemeinschaftsgeist erfordert, dass wir die Erde als unser gemeinsames Eigentum betrachten und behandeln, so gut wie Luft und Sonnenlicht. Warum tut Ihr es nicht? Ihr sagt: ,das tun die Wilden; aber Ackerbau, Kultur, ohne persönliches Eigentumsrecht ist unmöglich!'. Ich aber sage: gerade dieser Raub der Erde ist der erste und tiefste Grund menschlichen Elends, und so lange er besteht, ist die soziale Frage unlösbar. »

${ }^{11}$ Gleizes, Antoine, Thalysie; ou, La nouvelle existence, éd. L. Desessart, 1841.
} 
mais plus tôt d'un naturel cultivé. Les partisans comme Just de cette culture naturiste, même s'ils considéraient le naturisme comme naturel, n'envisageaient pas de le pratiquer dans le quotidien ou au travail. Il s'agissait dans la plupart de ces instituts de se dévêtir uniquement dans des espaces dédiés, des solariums aménagés, généralement entourés d'une haute palissade les protégeant des regards indiscrets. La pelouse était tondue, les visiteurs disposaient de douches et de cabines pour se changer et de chaises longues pour s'étendre. Souvent même, les sexes étaient séparés. Comme le souligne Faltin, le crédo du retour à la terre voulu par Just et d'autres n'était pas à prendre au pied de la lettre ; il ne s'agissait pas de rétablir un état de la nature archaïque mais de s'inspirer plus modestement de la nature pour mener une vie meilleure sans refuser les bienfaits de la modernité ${ }^{12}$.

L'historienne Judith Baumgartner fait aussi état à cette époque d'une grande activité associative dont témoignent les publicités, l'engouement pour les livres de cuisine et recettes végétariennes et la naissance des Reformhäuser ou magasins de produits naturels. Le Livre de Recettes Végétariennes (Vegetarisches Kuchbuch) d'Anna Springer fut ainsi réédité six fois entre 1900 et 1914. Les magasins de produits naturels naissent avant la fin du 19ème siècle, à l'instar de la Centrale pour la Santé (Gesundheitszentrale) créée par un commerçant de Berlin, Karl Braun, membre du Berliner Naturheilverein, une maison de vente par correspondance de produits naturels. À Leipzig, en 1888, Paul Garms fonde à son tour la Thalysia $\mathrm{GmbH}$, en référence à l'ouvrage du Français J.A. Gleizes. Celui-ci débute son activité avec un restaurant végétarien et se met à fabriquer des aliments végétariens pour ses 14 enseignes Thaylisa réparties principalement dans les grandes villes. Quelques années plus tard, en 1900, apparaît à Wuppertal une enseigne appelée Maison de Réforme Fontaine de Jouvence (Reformhaus Jungbrunnen). Le réseau de magasins de produits naturels commence à s'organiser, si bien qu'en 1909, 18 entrepreneurs décident de créer l'Association des Propriétaires Allemands de Magasins de Produits Diététiques (Vereinigung Deutscher Reformhausbesitzer). Ces magasins veillent à proposer selon les principes de la Lebensreform des aliments diététiques et naturels tels que des plantes médicinales, des boissons sans alcool, des noix et des fruits secs, du pain complet et certains produits sont exclusivement distribués dans ces enseignes. En parallèle, on assiste à l'ouverture de restaurants végétariens, le premier restaurant végétarien datant sans doute de 1871 à Bayreuth $^{13}$. A Berlin, Leipzig et à Vienne apparaissent au seuil du 20ème siècle des restaurants et des auberges végétariennes dont le nombre dans l'Empire de Guillaume II atteint 184 à la veille de la Première guerre mondiale. Comme le rappelle néanmoins Baumgartner, les cafés et les bars sans alcool, servant du cacao et de l'eau de Seltz, semblaient, à en juger par leur nombre, rencontrer plus de succès que les lieux refusant de servir de la viande ${ }^{14}$.

\section{Les coopératives et communautés végétariennes}

La Lebensreform, non dotée de moyens politiques de transformer la société, nourrissait cependant une ambition sociale. Le végétarisme était perçu comme un moyen d'établir un monde plus fraternel ne reposant pas sur des aliments d'animaux abattus. Certains réformateurs désirèrent de fonder des colonies ou cités-jardins dont le but premier était de parvenir à l'autosuffisance dans le respect de la nature. La Gemeinnützige vegetarische Obstbau Kolonie Eden ou Colonie d'Arboriculture Eden fut précédée de la création en 1887, à Vienne, de la Landkommune, regroupée autour du peintre symboliste végétarien, nudiste et pacifiste, Karl Wilhelm Diefenbach. Cette dernière servit de modèle quelques années plus tard à la communauté du Monte Verita. Des colonies végétariennes furent fondées en Autriche, Suisse, en Italie, en Grèce et même jusque dans l'île Floreana, au coeur des

\footnotetext{
${ }^{12}$ Faltin, Thomas, Heil und Heilung, Geschichte der Laienheilkunde und Struktur antimodernistischer Weltanschauungen in Kaiserreich und Weimarer Republik am Beispiel von Eugen Wenz (1856-1945). Diss. Franz Steiner Verlag, 2000, p. 151-152.

${ }^{13}$ C'est là où Richard Wagner, quelques années plus tard, en 1882, demanda à ce qu'on dresse une table végétarienne pendant les Bayreuther Festspiele. Cf. Baumgartner, Judith, Baumgartner, Judith (2001): Vegetarisch im 20. Jahrhundert - eine moderne und zukunftsfähige Ernährung. In: Vegetarismus. Zur Geschichte und Zukunft einer Lebensweise. Hg. v. Linnemann, Manuela und Schorcht, Claudia. Erlangen: Fischer S. 107 - 126. (Tierrechte-Menschenpflichten, Bd. 4)

${ }^{14} \mathrm{Cf}$. à ce sujet l'article de Baumgartner, « Besser essen, besser sein », in Zeit/Geschichte Nr. 2, 21.05.2013.
} 
Galapagos ${ }^{15}$. L'une des plus célèbres, la colonie Eden fut créée en 1893 dans un restaurant végétarien de Berlin-Moabit où se rassemblèrent 37 réformateurs proches des idées d'Eduard Baltzer. Le terrain, situé non loin d'Oranienburg fut acheté à bas prix et il devint bientôt d'usage de s'y déshabiller. Ses membres y bâtirent des maisons et y firent pousser des arbres fruitiers et des arbustes à baies. Des jardins furent d'abord créés puis distribués aux locataires sous la forme de baux. On y dénombrait pas moins de 50000 arbustes à baies, 15000 arbres fruitiers, 3000 noisetiers et 200000 fraisiers. Le lieu ne tarda pas à devenir un espace de production de jus et de confitures, dans le strict respect des principes du végétalisme. Au début, seuls les végétariens eurent le droit d'y construire des bâtiments et de s'y installer et l'élevage y fut strictement interdit, y compris pour les poules et les abeilles. Des fermes et des bâtiments résidentiels furent édifiés sur le terrain, préludes à la mise en place progressive d'une véritable entreprise commerciale, une coopérative également dotée d'une école et d'une imprimerie. Les lotissements furent bientôt complétés par l'édification d'une maison de repos et d'une maison d'hôtes. Des publicités vantant la «viande de substitution » en provenance de la colonie Eden furent imprimées. Les produits commercialisés par la coopérative Eden rencontrèrent un grand succès dans les magasins de produits naturels comme le «beurre de la Réforme Eden » à base de margarine végétale. Mais en raison de difficultés financières dues à un nombre insuffisant d'adhérents, l'Eden raya de ses statuts la mention « végétarien »- permettant à ceux qui ne l'étaient pas d'accéder aussi à la colonie - et finit par commercialiser du miel, un produit phare de la citéjardin, infléchissant en cela sa position strictement végétalienne en continuant cependant jusqu'à la fin des années 1920 à ne cultiver que des produits végétariens. Si le naturisme y fut pratiqué de même que l'abstinence en termes d'alcool et de tabac, l'usage de vêtements plus adaptés comme les sandales, des vêtements féminins légers rompant avec l'usage des corsets, des jupons ou des chapeaux fut répandu et participa d'une libération des contraintes corporelles, notamment chez les femmes. Durant la République de Weimar, la colonie se déclara proche des idées du Troisième Reich, ce qui permit à la colonie de continuer son existence sans être inquiétée par les autorités nationalsocialistes ${ }^{16}$.

La colonie végétarienne du Monte Verita ${ }^{17}$ va, en termes de pratiques naturistes, au-delà de la colonie Eden où il ne semblait n'être pratiqué qu'à la marge. Le choix du Monte Verita, au bord du Lac Majeur, est en partie dû à l'ouverture du tunnel du Gothard en 1882 qui rendit plus facile l'accès au Tessin depuis le nord. Comme le rappelle Harald Szeemann, «Ascona, dans les années 1990, était l'avant-poste le plus au sud d'une profonde Lebensreform venue du nord ${ }^{18}$. Fondée par le fils d'un grand industriel antwerpois, Henri Oedenkoven, et sa compagne, Ida Hofmann. Oedenkoven, souffrait de problèmes articulaires et de maux d'estomac. Il rencontra Ida Hofmann lors d'une cure dans l'institut d'héliothérapie de Rikli à Bled (Slovénie) et adopta une cure végétarienne. Le végétarisme prôné par Oedenkoven va de pair avec un discours sur «l'hygiène naturelle », la consommation de viande nuisant selon lui durablement à la santé, contrairement aux légumes (non

\footnotetext{
${ }^{15}$ Un dentiste allemand, Friedrich Ritter et sa compagne Dore Strauch, végétariens convaincus, décidèrent de quitter la société bourgeoise et de fonder une colonie végétarienne, isolés, sur l'île Floreana, dans les Galapagos. Suivirent d'autres colons dont une fantasque et mégalomane baronne venue avec ses deux amants et autoproclamé «impératrice de Floreana ». Son corps disparaîtra dans la fameuse histoire des Galapagos. Ritter, lui, serait mort quelques années plus tard pour avoir mangé une poule morte, la sécheresse ayant entamé les récoltes. Cf. Wittmer, Margrett, Floreana, Floreana: A Woman's Pilgrimage to the Galapagos (Englisch) Taschenbuch - 29. Oktober 2013. Lire à ce sujet: http://www.laurefred.com/laffaire-des-galapagos/

${ }^{16}$ Mosse, George, Die völkische Revolution. Über die geistigen Wurzeln des Nationalsozialismus. Frankfurt/Main 1991, p. 123.

${ }^{17}$ Noschis, Kai 2011, Monte Verità : Ascona et le génie du lieu, Lausanne, PPUR
}

${ }^{18}$ Szeemann, Harald, Monte Verita - Berg der Wahrheit, in: Gabriella Borsano Claire Halperin, Harald Szeemann (Hrsg.): Monte Verità - Berg der Wahrheit: lokale Anthropologie als Beitrag zur Wiederentdeckung einer neuzeitlichen sakralen Topographie. Armando Dadò u. Electa Editrice, Lugano u. Mailand 1978, p. 6 (Katalog zur Ausstellung in der Casa Anatta, Monte Verità; Gemeindemuseum, Ascona; Marianne-von-Werefkin-Stiftung, Gemeindemuseum, Ascona; Neue Turnhalle, Collegio Papio, Ascona; Ehemaliges Theater, Collegio Papio, Ascona, 8. Juli bis 30. August 1978; Brissagoinseln, 8. Juli bis 27. August 1978. 
cuits) qui fortifient le corps. $\mathrm{Nu}$, par son exposition directe au soleil et au vent, le corps s'aguerrit. Oedenkoven avait aussi suivi les préceptes de Louis Kuhne (1835-1901), un des fondateurs de la naturopathie. Il avait séjourné à plusieurs reprises au sein de son sanatorium végétarien de Leipzig dont il sortit guéri, sans recours aux médicaments mais en adoptant une hygiène naturelle. Kuhne préconisait en cas de maladie des bains de siège à l'eau froide et la consommation de fruits crus mais aussi de noix, de céréales et de blé complet.

En union libre avec Hofmann, Oedenkoven décida avec la fortune familiale de fonder près d'Ascona une colonie sur le Monte Verita, inspirée de la Landkommune. Le «végétabilisme » que mentionne plus haut Hermann Hesse correspond à une forme de végétarisme radical que l'on nommerait aujourd'hui le végétalisme. Le couple se qualifiait lui-même de «végétabiliste » et renonçait à tout produit d'origine animale, y compris au lait, aux oeufs et au miel. Ils ouvrirent sur le Monte Verita un sanatorium où les principes du végétalisme furent scrupuleusement appliqués ${ }^{19}$. L'écrivain Erich Mühsam, qui visita la « coopérative végétabilienne », tourna légèrement en dérision ${ }^{20}$ au détour d'une chanson ce qui fut ironiquement baptisé le « saladorium » :

On mange de la salade, oui, on mange de la salade

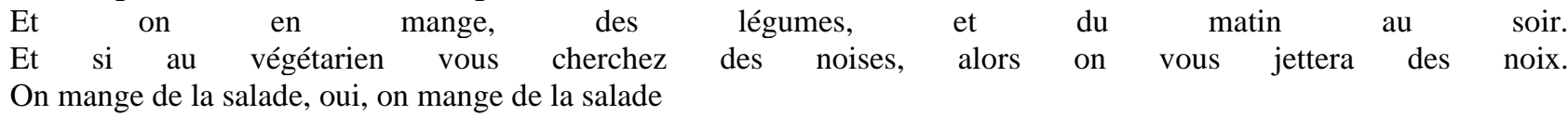

Et on en mange, des légumes, et du matin au soir. 21

L'expérience végétarienne de Mühsam qui résida au Monte Verita en 1904 ne semblerait pas avoir enchanté les papilles de l'écrivain, celui-ci déclarant dans une lettre : «Je suis au bord du Lago Maggiore où, vêtu d'un pittoresque costume de lin, je déambule en pantacourt, pieds nus et le poitrail débraillé - et je vis en végétarien - c'est-à-dire sans odeur et sans goût - vivant. Tout cela pour le bien de ma santé. ${ }^{22}$ Cette coopérative végétarienne devait être envisagée comme une critique de la modernité, de l'accélération, de la civilisation éloignée du rythme de la nature. Dans cette communauté, naturisme et végétarisme sont les deux mamelles de la Lebensreform souhaitée par les fondateurs du Monte Verita. Si le naturisme n'était pas une pratique partagée par tous, certaines femmes et hommes préférant à la nudité totale déambuler dans d'amples tuniques blanches, l'expérience du végétarisme combiné à la nudité fait partie intégrante du programme de libération corporelle voulu par les réformateurs. C'est dans les forces de la nature que le couple OedenkovenHofmann, accompagné des frères Gusto et Karl Gräser, espère trouver le salut et la vérité. Au début de la Coopérative Végétarienne, il n'y avait ni électricité, ni eau courante. Le but de la communauté fut d'abord de parvenir à l'autosuffisance. On y planta des arbres fruitiers, des châtaigniers et des palmiers et le Monte Verita ne tarda pas à attirer des anarchistes, des artistes, des écrivains, des intellectuels, des anthroposophes, des théosophes et des adeptes en général d'une médecine naturelle, sans médicaments et d'un mode de vie alternatif ${ }^{23}$. Les résidents y effectuaient des travaux du jardin et du potager dont ils consommaient les fruits et les légumes, construisirent des huttes d'air et de lumière, se baignaient nus ou prenaient des bains de soleil dans les solariums prévus à cet effet protégés des regards par des palissades - , ce qui leur valut le surnom de «balabiott» qui dans le dialecte lombard signifie « ceux qui dansent nus ».

La communauté du Monte Verita, comme dans la cité-jardin Eden, préconisa de délaisser les corsets et les vêtements rigides prévalant à l'époque au profit d'habits confortables. Cette libération

\footnotetext{
${ }^{19}$ Schwab, Andreas, Monte Verrat - Sanatorium der Sehnsucht, Zürich, 2003, p. 78.

${ }^{20}$ Mühsam, Erich, Aus Ascona. Eine Broschüre. Locarno (Verlag von Birger Carlson) 1905.

${ }^{21}$ Op. cit. : «Wir essen Salat, ja wir essen Salat / Und essen Gemüse von früh bis spat. /Und schimpft ihr den Vegetarier einen Tropf / So schmeissen wir euch eine Walnuss an den Kopf. /Wir essen Salat, ja wir essen Salat / Und essen Gemüse von früh bis spat. »

${ }^{22}$ Op. cit. : « Ich bin ,am Lago Maggiore, wo ich, mit einem malerischen Leinenkostüm angetan, kurzhosig, barfüßig und nackthalsig herumstelze und vegetarisch - das heißt geruch- und geschmacklos - lebe. Dies alles von wegen der Gesundheit. »

${ }^{23}$ Le Monte Verita vit passer comme hôtes outre Hermann Hesse, Kropotkine, Rudolf Steiner, les danseuses Isadora Duncan et Mary Wigman, Ruldof Laban, C.G. Jung, Otto Gross, Franz Kafka...
} 
corporelle, motivée par une quête de la santé, passe par une mise à nue du corps en plein air et un régime alimentaire naturel, caractérisé par la consommation de fruits et de légumes (crus), où la consommation de sels, de thé, de café, de tabac et d'alcool est proscrite. Si naturisme et végétarisme sont pratiqués ensemble, c'est parce que la Lebensreform repose sur une approche holistique du corps. A partir des années 1920, la sanatorium du Monte Verita connut des problèmes d'argent conjugués à des divergences d'opinion, les uns comme Oedenkoven étant favorables à un minimum de confort et au développement de structures d'accueil pour curistes, prêts à faire des concessions avec la modernité, les autres plus radicaux, refusant le confort et préférant le troc à l'argent, à l'instar du poète-vagabond Gusto Gräser - que l'on retrouve sous les traits du personnage Léo dans Le Voyage en Orient de Hesse. Déçu par la tournure prise par l'établissement, Gräser se retira en ermite dans une grotte, un peu plus bas en amont. C'est pour des raisons éthiques que Gräser était végétalien et refusait la souffrance des animaux au même titre que toute forme de violence. Le végétalisme et l'abstinence ne s'imposèrent pas de soi dans la communauté. Au début, il n'était pas rare qu'Oedenkoven tombe sur des restes de fromage de brebis dans la communauté ou que les curistes ne descendent dans les villages voisins pour y consommer secrètement les vins du Tessin et du salami. Oedenkoven tenta d'abord de sauver son entreprise de la faillite en faisant appel à un apôtre du jeûne, un dénommé Arnold Ehret, qui tout comme lui, aurait guéri de la maladie de Bright (inflammation des reins) par un régime à base de légumes crus et un jeûne record de presque cinquante jours. Pour Ehret, le jeûne était une condition indispensable pour se débarrasser des toxines et de l'acide urique produite par l'alimentation carnivore car les viandes selon lui se décomposent dans l'organisme et sont sources de maladie. Aussi, en 1907, Ehret introduisit au Monte Verita son école de diététique à base de légumes crus que fréquenta également Hermann Hesse, avant qu'Ehret n'émigre en 1914 aux Etats-Unis pour y diffuser ses théories sur l'hygiène naturelle. Mais les dettes s'accumulant, les préceptes du végétalisme strict furent assouplis à l'intérieur de la communauté et de la viande finit par être servie au menu pour les curistes, afin d'attirer un plus grand nombre de visiteurs.

Un intéressant reportage consacré aux « naturistes du Monte Verita », qualifiés de « peuplade blanche dans une forêt des bords du Lac majeur », fut rédigé en 1907 par le journaliste Jules Chancel pour le journal l'Illustration. Celui-ci livre des informations sur les pratiques végétariennes et naturistes du Monte Verita. Chancel dresse le constat suivant: «Il parait que tout individu qui a été initié aux secrets de l'hygiène naturelle et du végétarianisme subit une sorte d'évolution qui modifie sa manière de concevoir la vie. » Au sujet de la nudité pratiquée sur place, voici ce que note Chancel:

Bien entendu, pas de corsets, pas de jupons, bras, jambes, et cou découverts. Les cheveux tombent sur les épaules ou sont retenus par un simple ruban. Encore une fois, le costume, comme le reste, est facultatif, et ceux qui veulent conserver nos modes habituelles ont le droit de le faire ; mais, au bout de quelques semaines de séjour, tout le monde en arrive forcément et logiquement à se vêtir le moins possible. La coquetterie disparaît, la pudeur s'abolit devant la simplicité pure de la nature bienfaisante. ${ }^{24}$

Les naturistes semblent d'après le témoignage de Chancel prendre leur repas végétarien selon un rituel bien établi, non sans rappeler leurs ambitions hygiénistes ${ }^{25}$ :

Chaque naturiste a son tiroir. Il entre, il consulte le menu dont nous donnons plus loin un spécimen avec les prix fort abordables, et il écrit sur une feuille de papier les mets qu'il désire: Cette feuille est glissée dans une boite et, quelques minutes après, on va ouvrir son tiroir. Sur un plateau sont disposés les ustensiles et les différentes petites soucoupes en aluminium contenant la bouillie de froment, le pain complet, les fruits qui constituent la nourriture végétarienne. Ah ! dame ! je ne vous affirmerai pas que cette nourriture flatte énormément le palais, mais les initiés vous diront d'abord qu'il ne faut pas exciter l'appétit, car c'est ainsi qu'on arrive à se nourrir trop, ensuite qu'ils trouvent, eux, des jouissances très grandes en dégustant ces mets peu engageants pour les profanes. D'ailleurs, il ne faut pas croire qu'elle soit simple, cette

\footnotetext{
${ }^{24}$ Chancel, Jules, L'Illustration, 27 juillet 1907.

${ }^{25}$ Chancel, Jules, L'Illustration, 27 juillet 1907, « Il me reste encore à établir la mentalité des naturistes avec lesquels il m'a été donné de causer. Ces gens sont évidemment différents de ceux que l'on rencontre dans la vie ordinaire. Il se dégage d'eux quelque chose de cette douceur et de cette foi mystiques que l'on trouve dans les couvents. Et cependant, je le répète, aucune idée de religion ou de secte n'existe dans leur réunion. Ce sont simplement des hygiénistes qui se défendent, même d'être des philosophes. »
} 
cuisine végétarienne. On doit la préparer dans des marmites spéciales, aptes à concentrer tous les sels nutritifs des légumes ou des fruits que, dans notre cuisine habituelle, nous laissons perdre par l'évaporation, ou que nous noyons dans l'eau. Les cuisiniers végétariens ne se servent jamais d'eau. C'est l'eau du fruit ou du légume qui doit seule aider à sa cuisson. Mais voici le repas terminé. Il est court. Les naturistes qui sont allés manger, où bon leur semblait, le contenu de leurs plateaux, les rapportent eux-mêmes dans un bassin d'eau courante où les récipients se nettoient facilement. ${ }^{26}$

On peut, au regard des écrits et des témoignages de l'époque, se demander si, finalement, le végétarisme prôné au Monte Verita, ne provenait pas davantage de considérations hygiénistes portant ses résidents à s'intéresser aux bénéfices du végétarisme sur leur santé avant tout plutôt que de considérations éthiques relatives à la souffrance infligée aux animaux (idées toutefois présentes mais chez des personnes plus radicales comme Gusto Gräser). La période strictement végétalienne du Monte Verita fut interrompue en 1920 quand les dettes commencèrent à s'accumuler et que pour attirer davantage de visiteurs, il fallut bientôt servir de la viande pour satisfaire leurs besoins, avant qu'Oedenkoven et Hofmann n'émigrent en Espagne puis au Brésil pour y fonder une nouvelle communauté végétarienne. Mais ce ne furent pas les seuls à s'exiler et à diffuser leurs idées. Ehret était déjà parti aux Etats-Unis en 1914 où il devint un pionnier de l'hygiène naturelle et ses idées furent reprises dans l'Amérique alternative des années 1970-1980 à l'instar du best seller Fit for Life de Harvey et Marylin Diamond ${ }^{27}$ basé l'hygiène de vie et un régime sans viande, à base de fruits et de légumes crus. Les idées des naturistes et végétaliens allemands servirent d'inspiration dans les milieux alternatifs nord-américains intéressés par la thématique du retour à la terre mais elles exercèrent aussi une influence certaine en France.

\section{L’influence du végétarisme et du naturisme allemand à Paris}

La nudité, l'ascèse et la gymnastique ${ }^{28}$ sont avec les naturistes des techniques expérientielles qui transforment le vécu corporel. Le naturisme, autrefois Gymnosophie, est né en France, avec le géographe Élisée Reclus (1830-1905) ${ }^{29}$ qui y voyait en 1866 à la fois un moyen de revitalisation physique, un rapport au corps complètement différent de l'hypocrisie et des tabous qui sévissaient alors, une conception plus conviviale de la vie en société, et une incitation à respecter la planète. Notons qu'Elisée Reclus, précurseur de la pensée écologiste, fut aussi l'un des premiers défenseurs de la cause animale dans un écrit les raisons de son aversion pour la viande et son choix "légumiste » pour un végétarisme strict. ${ }^{30}$ Hugo Höppener dit Fidus (1868-1948) ${ }^{31}$ crée en 1894 une « prière de lumière » (un jeune homme qui étire ses bras dans le ciel) qui devient le symbole de cette culture du corps. Le sociologue Heinrich Pudor (1865-1943) est considéré comme « le père de

\footnotetext{
${ }^{26}$ Op. cit.

${ }^{27}$ Diamond, Harvey and Marilyn, Fit for Life (1985)
}

\footnotetext{
${ }^{28}$ Peeters, Evert, 2006, Authenticity and Asceticism: Discourse and Performance in Nude Culture and Health Reform in Belgium, 1920-1940 Journal of the History of Sexuality, Volume 15, Number 3, July 2006, pp. 432-461 Michael Hau, The Cult of Health and Beauty in Germany: A Social History 1890-1930 (Chicago: University of Chicago Press, 2003), 82-100, 150-75; Chad Ross, Naked Germany: Health, Race and the Nation (Oxford: Berg, 2005), 1-15, 135-60.

${ }^{29}$ Reclus E., 1866, « Du sentiment de la nature dans les sociétés modernes », in La Revue des deux mondes, $n^{\circ} 63,15$ mai 1866, pp.352-381.

${ }^{30}$ Reclus, Elisee, A propos du végétarisme, Elisee, 1901.

${ }^{31}$ Janos Frecot, Johann Friedrich Geist, Diethart Kerbs, Fidus, 1868-1948: Zur ästhetischen Praxis bürgerlicher Fluchtbewegungen (Munich: Rogner und Bernhard, 1972), 9-12, 15-24;
} 
la culture de corps $»^{32}$. C'est sous le nom d'emprunt Heinrich Scham qu'il a publié plus de 110 livres. Dès 1893 il avait publié à Dresden-Loschwitz un pamphlet intitulé Nackende Menschen. Jauche der Zukunft (Les Hommes nus. Un cri triomphant lancé au futur), et inventera en 1906 le mot Nacktkultur (culture du nu) dans son Katechismus der Nacktkultur. Dans le Guide pratique de l'Education physique, publié en 1909, G. Hebert soutient cette méthode d'entraînement à résistance au froid et aux intempéries qui " se fait tout naturellement en travaillant le torse nu, au moyen de bains d'air en toutes saisons ${ }^{33}$. Dans Le code de la force en 1910, Georges Hébert (1875-1957) estime que le dévêtissement pudique à des fins d'endurcissement est la condition nécessaire pour les bains d'eau d'air et de soleil, s'opposant ainsi à ceux qui « redoutaient l'exercice par crainte de l'effort, l'action vivifiante du grand air, la chaleur même du soleil $»^{34}$. Le Marquis Melchior de Polignac (1880-1950), dans son parc Pommery à Reims, crée une terrasse réservée aux athlètes à demi nus ${ }^{35}$. En 1913, G. Hebert, directeur dans le Parc de Pommery, organise la gestion des bains d'air, de soleil, de lumière, l'hydrothérapie, les massages, l'exercice physique, les bains dans la piscine l'hiver, et les bains de neige. Pas de mixité car les femmes ont un stade particulier. Une application de la méthode naturelle est réalisée dans 33 écoles primaires et lycées de la ville de Reims. Il s'agit d'une méthode simple, à la base de laquelle se trouvent la marche, la course, le saut, le grimper, le lever, la natation et les exercices de défense naturelle. Le Parc Pommery offre déjà toutes les installations nécessaires pour la bonne marche de ce collège, qui suscite cependant quelques polémiques, certains intellectuels étant choqués par l'accouplement du mot collège au mot athlètes, d'autres qualifiant ce collège de " boutique à muscles ", de " boîte à bachot pour candidats aux Jeux olympiques ». Mais la nouvelle méthode séduit beaucoup de jeunes qui sont rebutés par les systèmes fastidieux d'éducation physique pratiqués dans les écoles. En six mois, une piste ovale, un gymnase couvert de 40 mètres sur 20 et une piscine en plein air sont construits, accompagnés de salles de boxe et d'escrime.

En 1913 le camp d'entraînement de La Palestra ${ }^{36}$, dépendance du collège gymnique féminin et enfantin, est fondé aux abords de la plage de Deauville sur Mer dans le Calvados. Le régime de vie est rustique, sous tentes comme " une école de culture mentale autant que culture du corps ${ }^{37}$. La description classique du caractère nerveux de la jeune fille trouve dans le manque d'activité physique la cause d'une vie trop sédentaire et trop artificielle : « C'est par le contact constant du grand air, par les bains d'air et de soleil autant que par l'exercice, enfin par l'alimentation la plus frugale et la plus naturelle possible que l'organisme se débarrasse de toutes ses impuretés et qu'il augmente ses défenses naturelles contre la maladie. »

\footnotetext{
${ }^{32}$ Cluet, Marc, 2000, La « libre culture ». Le mouvement nudiste en Allemagne depuis ses origines au seuil du XXe siècle jusqu'à l'arrivée de Hitler au pouvoir (1905-1933). Présupposés, développements et enjeux idéologiques, Université Paris-Sorbonne-Paris IV.

${ }^{33}$ Hebert G., 1909, Guide pratique d'Education physique, Paris, Vuibert, p. 8.

${ }^{34}$ Hebert G., 1910, Le code de la force, Paris, Vuibert, p. 91.

35 Tissié, P. (Dr), 1914, L'homme nu. La Méthode Naturelle et le Collège d'Athlètes de Reims. Critique physiologique, pédagogique et médicale, RJSHS, 17-49 et 55-59.

${ }^{36}$ Meden-Philippe, Pierre, 2019, "Hebertist Palestra: Physical Education for Women, Leisure and Natural Lifestyle", Educação em Revista

${ }^{37}$ Hebert G., 1922, La palestra. Camp d’Entraînement pour Jeunes Filles de 15 à 21 ans, Paris, Vuibert, p. 6.

Voir aussi Hébert, G. 1925, Une institution originale - les palestres d'hiver et d'été du collège gymnique La Palestra, Paris, Vuibert.
} 
Les migraines, les insomnies, la constipation et « tous les maux particuliers au sexe féminin, disparaissent comme par enchantement après une cure suffisante de plein air et d'exercices ${ }^{38}$. Fortifier la santé, développer les muscles, entraîner le corps, endurcir l'organisme, simplifier la vie, développer le sens pratique sont les buts recherchés dans ce camp, le corps ne suffisant pas à lui seul : restant " en plein air toute la journée et se couvrant le plus sommairement possible ${ }^{39}$, les jeunes filles éduquent autant le corps et l'esprit par la recherche d'une harmonie et d'un équilibre. En évitant et le surmenage et le relâchement ou la paresse, l'entretien en bonne santé est assuré " par la vie continue à l'air libre $»^{40}$. Le bondissement sur les mains, la lutte corps à corps, la course de vitesse, la dissimilation à l'indienne, le ramper, "le jeu américain à la mode $"{ }^{41}$ une partie de basket-ball, le tir à l'arc, les exercices de canotage et de natation, un geste de danse s'organisent autour de différents stades : le stade de danse, le stade des jeux, le grand stade d'entraînement. En 1920, Adolf Koch (1896-1970), l'enseignant berlinois, obtient le droit de faire pratiquer à ses élèves des cours d'éducation physique en nu intégral en fondant "l'École des nudistes " avec une gymnastique spéciale, présentée publiquement jusque dans les années 30 au Berliner Schauspielhaus. II publie en 1929 « Nudité, culture du corps et éducation ».

\section{Végétalisme et anarchisme}

Mais les naturiens anarchistes, à la différence des naturistes aussi intégristes, engagent leur corps aussi dans un retour économique à la Terre. Végetalisme et anarchisme en action se sépareront du naturisme par un retour à une vie sans contact avec la civilisation. Les naturiens, courant apparu en 1874 à Paris, ont l'idée de retrouver en cultivant la terre dans son état naturel et intégral : pour cela la vie de l'individu dans la forêt doit se réaliser avec un minimum d'industrie individuelle. Les personnalités dominantes ce végétarisme ${ }^{42}$ dans les milieux naturiens furent Georges Butaud (1868-1926), sa compagne Sophia Zaïkowska (1875-1939), et Louis Rimbault (18771949). Cette nouvelle orientation fut qualifiée par les naturiens d'origine de "néo-naturianisme " et ses tenants de " néo-naturiens », selon un terme créé par Henri Zisly (1872-1945) en 1913. Avec son ouvrage Terre libérée, Louis Rimbault (1877-1949) fit du végétalisme un projet communautaire : il veut créer « une œuvre sérieuse et durable, sans tendance, consacrée à l'étude de la libération individuelle la plus entière et la plus immédiate ${ }^{43}$. Avec les soutiens du Dr Legrain, médecinaliéniste, du Paul Carton (1875-1947), de Jacques Demarquette (1888-1969), de Marcel Labbé44, de Jean Morand ${ }^{45}$ ou de Charles Richet, Rimbault dirigea à partir de 1924 une colonie agricole autarcique, pratiquant le végétalisme, à Luynes, en Indre-et-Loire, du nom de Terre Libérée.

La purification par le régime végétaliste suppose des bains de siège, de soleil, d'eau courante, « la vie laborieuse en plein air (le torse et les jambes nus), quelques heures de marche, quelques

\footnotetext{
${ }^{38}$ Op. cit., p. 8.

39 Op. cit., p. 11.

${ }^{40}$ Op. cit., p. 28

${ }^{41}$ Op.cit., p. 36.

${ }^{42}$ www.vegetarisme.fr. André Mery pour L'alliance Végétarienne a réalisé une histoire très précise sur la base d'archives de ce mouvement sous le titre « Eléments d'histoire du végétarisme en France », 1998, Cahier n², 20 p.

${ }^{43}$ Rimbault L., "La Terre Libérée", Le Néo-Naturien, n 14, octobre-novembre 1923.

${ }^{44}$ Chef de laboratoire à la clinique Laennec, auteur avec son frère et Louis Landozy d'une étude sur l'hygiène alimentaire des ouvriers parisiens (1905). En relation avec la Société Végétarienne de France.

${ }^{45}$ Secrétaire général de la Société Végétarienne de France.
} 
bonnes courses les cheveux au vent ${ }^{46}$; l'alimentation est une purgation par les jus de fruits produits par soi-même et le bouillon de céréales reminéralisant le corps. Se soigner naturellement par une médecine biologique vise à faire disparaître les vices et les défauts, la guérison des infirmités psychiques, psychiques ou mentales. La mise au point de la basconnaise, selon une recette de Clémence Rimbault, est composée « de variétés végétales alimentaires, potagères ou sauvages, crues de préférence et cuites pour certaines raisons d'adaptation ${ }^{47}$. Ce végétalisme, plutôt qu'un végétarisme qui serait une privation du lait « indispensable ${ }^{48}$, sélectionne seulement les produits biologiques de la "Terre libérée ». Ce texte de Louis Rimbault précise encore davantage cette séparation entre végétalisme et naturisme : « Le mouvement végétalien [...] prend en France et un peu partout à l'extérieur une importance sérieuse. Le naturisme cherche à endiguer le mouvement végétalien qui est "non coopérateur " aux forces du mal sur lesquelles reposent l'Etat et le naturisme sanctionne les situations acquises, le naturisme n'engage à rien et le politicien y foisonne. Nous, végétaliens, sommes sabotés et nos articles refoulés, nos publications renvoyées. Nous sommes pour ces gens là des " anarchistes en action " Ceux-là ont vu clair ! " ${ }^{49}$ Le naturisme voudrait intégrer le végétalisme comme un moyen et non comme une fin en soi du retour à la terre. Le type de communauté proposé par les naturistes est une mise entre parenthèses dans des enceintes réservées avec des entrées privatives, séparant les sexes et les cartes de fidélité. Le végétalisme anarchique est un lieu communautaire et non un club privé, une association de membres sélectionnés.

\section{Le nudisme Kienné de Mongeot}

En se référant à la libre culture, Kienné de Mongeot (1897-1977) a pu être un précurseur du nudisme en France, établissant un centre de nudité intégrale (Wolga, 2018 : 45), le Sparta club , qu'il défendit à travers ses revues et publications. En 1929, au sein de son Sparta Club, un solarium gymnique fut ouvert dans le parc du château de Garambouville dans l'Eure. Puis il fut déplacé au Douaire près de Gaillon, et au manoir Jan à Fontenay-Saint-Père à $6 \mathrm{~km}$ de Mantes et enfin au Chateau d'Aigremont, par Chambourcy. Le Sparta Club a été d'abord, sous l'égide du frère de Kienné, Gilbert de Mongeot, une salle au 3 rue de Logelbach « dans un des quartiers les plus sains de Paris " pour un « cours de culture physique. Traitement de l'obésité, de l'insuffisance respiratoire et musculaire. Hydrothérapie complète gratuite. Contrôle médical sur demande ». Les corps n'y sont pas mixtes et la tenue pour les messieurs est le slip et pour les dames " slip et cache-seins ou maillot ». En même temps, comme l'illustration publicitaire le montre, un " centre gymnique et de Lumière du "Sparta Club ", sorte d'annexe aéré hors de Paris, est proposée "située dans une contrée riante, sur un plateau permettant les cures d'air et de repos à 1 h20 de Paris, parc boisé de 15 hectares, entièrement clos ». Cette précision sur la clôture signifie, comme dans les clubs allemands entièrement fermés, que la nudité intégrale sera possible même si cela n'apparait pas. Le public ne doit pas être des athlètes mais au contraire « ce sont les déficients qui nous intéressent

\footnotetext{
${ }^{46}$ Rimbault, Louis, Les Secrets bienfaits de la maladie : les soins exécutant médecine et médecins, ce que le visage révèle. — Luynes : Terre libérée, s.d. — 80 p, ici p. 28.

${ }^{47}$ Op.cit., p. 47.

48 Végétarisme ou Végétalisme?, Rimbault, Louis, Les Secrets bienfaits de la maladie : les soins exécutant médecine et médecins, ce que le visage révèle. — Luynes : Terre libérée, s.d. — 80 p, ici p. 50.

${ }^{49}$ Lettre de Rimbault à Armand, 16 février 1930, I.F.H.S., Fonds Armand, 14 AS 211.
} 
plus particulièrement » (De Mongeot, 1932a : 3), ceux qui voudront bien progresser dans la pratique par une réelle conviction morale. Mais ce travail pionnier en libre culture, qu'il a conduit pendant plus de quarante ans avec le Sparta club et ses publications, repose sur une ignorance du nudisme allemand. Kienné de Mongeot écrit dans son livre inédit La révolte des sexes ${ }^{50}$ :

En 1926, j'ignorais tout du mouvement nudiste allemand. Mais je pratiquais, le l'ai dit, les bains d'air et de soleil en état de nudité complète, presque depuis ma naissance [...] En 1926 ou 1927, les méthodes nudo-naturistes furent implantées en Suisse par un véritable apôtre, Werner Zimmermann. II leur consacra toute son existence. Sa mort en pleine activité physique et intellectuelle, à l'âge de plus de quatre-vingt-quatre ans, démontre l'excellence de ses méthodes. Il eût pour principal collaborateur, Eduard Fankhauser, qui dirige toujours un centre situé au bord du lac de Neufchâtel. Eduard Fankhauser a fondé et dirige une revue illustrée intégralement. C'est la meilleure revue du genre que je connaisse. Cette revue, interdite en France, est en vente absolument libre en Suisse, où le gouvernement fédéral, très sévère en ce qui concerne la morale, se montre extrêmement compréhensif à l'égard de la propagande, si elle est sérieuse, faite en faveur du nudo-naturisme. J'ajoute que Werner Zimmermann, fort de son expérience, se montrait très ouvert à la recherche d'une nouvelle et indispensable éthique sexuelle. C'est également en 1926, peut-être même plus tôt, que fut créé le Monte-Verita ».

\section{Conclusion}

Par sa méconnaissance de la date de la création du Monte Verita, Kienné de Mongeot donne à penser que si l'Allemagne a joué un rôle important de passeur du naturisme et du végétarisme strict en France, la France, à l'instar d'Elisee Reclus, sut développer aussi sa propre voie. Mais ce qu'il ressort des sources historiques, ce sont aussi des parallèles évidents et notamment des lignes de fracture, en France comme en Allemagne, entre un végétarisme et naturisme cultivé, ouvert aux petits arrangements avec la modernité, d'une part, et un végétalisme et un naturisme radical, anarchiste, adepte d'un retour à la nature, moins fantasmé mais plus difficilement applicable.

Ces différents passages du modèle du végétalisme au végétarisme en passant par le nudisme révèlent combien chaque communauté en Allemagne et en France aura défendue des idéologies différentes de la nature.

Selon un degré de purification et d'immersion dans la nature, la nudité ne touche pas simplement le corps physique mais aussi le dénuement métaphysique par un contact plus exalté aux éléments, la terre devant faire corps avec le naturiste: diététique végétalienne, économie minimales, coopérative sociale et hygiénisme purificateur.

\footnotetext{
${ }^{50}$ KIENNÉ de MONGEOT Marcel, La révolte des sexes, manuscrit inédit, 1977 (Fonds ANET Michel Pivert), p. 132.
} 